\title{
MODELING STUDY OF LASER BEAM SCATTERING BY DEFECTS ON SEMICONDUCTOR WAFERS
}

\author{
Srikumar Sandeep ${ }^{1}$ and Alexander Kokhanovsky ${ }^{2}$ \\ ${ }^{1}$ Ecole Polytechnique, Montreal, Canada \\ ${ }^{2}$ University of Bremen, Germany
}

\begin{abstract}
Accurate modeling of light scattering from nanometer scale defects on Silicon wafersiscritical for enabling increasingly shrinking semiconductor technology nodes of the future. Yet, such modeling of defect scattering remains unsolved since existing modeling techniques fail to account for complex defect and wafer geometries. Here, we present results of laser beam scattering from spherical and ellipsoidal particles located on the surface of a silicon wafer. A commercially available electromagnetic field solver (HFSS) was deployed on a multiprocessor cluster to obtain results with previously unknown accuracy down to light scattering intensity of $-170 \mathrm{~dB}$. We compute three dimensional scattering patterns of silicon nanospheres located on a semiconductor wafer for both perpendicular and parallel polarization and show the effect of sphere size on scattering. We further computer scattering patterns of nanometer scale ellipsoidal particles having different orientation angles and unveil the effects of ellipsoidal orientation on scattering.
\end{abstract}

\section{KEYWORDS}

Defect, Laser, Scattering, Semiconductor, Wafer, Gaussian beam, HFSS

\section{INTRODUCTION}

Leading-edge semiconductor integrated circuits have entered the $14 \mathrm{~nm}$ technology node, there bycontinuing the trend of producing smaller, faster, cheaper, and energy efficient chips [1]. Whilethis trend unquestionably stands out as one of the key enablers of the information age, it must berecognized that continuing this trend is becoming increasingly challenging because of the difficulty infabricating increasingly complex nanometer scale layers reliably and in a cost effective manner. Asfeature sizes continue to decrease in semiconductor chips, low manufacturing yield becomes a criticalproblem. Yield losses are caused due to imperfections or defects that could occur in any of the hundredsof manufacturing process steps that transform a bare Silicon wafer into a modern integrated circuit.Stringent process controls are required to ensure that each process step creates a desired result withinestablished tolerances. Optical and electron based wafer inspection tools play a crucial role in processcontrol by providing much needed feedback on a previously performed process step. The feedbackincludes critical information on the number, sizes, shapes, and material properties of defects - muchneeded information that can be used to adjust process steps to eliminate those defects, especially theones that are likely to affect yield. Finding and eliminating such yield-affecting defects as soon as theyoccur is imperative to limit yield loss, which otherwise may rapidly propagate to a large number ofwafers in a high-speed modern semiconductor fab. 
In the current $14 \mathrm{~nm}$ leading-edge technology node, reduced yield can often be correlated to the inability of current inspection tools to effectively detect defects that are smaller than $20 \mathrm{~nm}$. Novel fabrication processes used in leading-edge nodes result in an increased defect density as well new typesof defects. New materials such as strained silicon, high-k dielectrics, and III-V compounds improvetransistor performance, but create new defects due to their atomic scale interactions with Silicon.Further, new three-dimensional (3D) transistor designs such as FinFETs and new 3D stacking methodssuch as Through Silicon Vias (TSVs) require complex fabrication processes that often lead to addeddefects [3]. Enabling these advances require the development of improved defect inspection systems withultra-high sensitivity and throughput [1,2]. An understanding of light scattering by nanometer scaledefects located on a semiconductor wafer is important in the design and development of such inspectionsystems [4]. Such an understanding requires accurate modeling of optical scattering by semiconductordefects on or under the wafer surface.

A survey of previous modeling work related to this problem can be found in [4,5]. Analytical methodssuch as Mie theory [6] can only be used for scattering due to isolated spherical particles. Mie theory doesnot take into account the effect of surface beneath the scattering particle. Previously published worksinclude the application of approximate methods such as Discrete Dipole Approximation (DDA) [7, 8] fordefects of radius larger than $100 \mathrm{~nm}$ when excited with plane wave of longer wavelengths (e.g. $532 \mathrm{~nm}$ ).Furthermore, these analytical methods cannot be used for defects under the wafer or multilayer wafersubstrates. Hence there is a need to perform accurate full wave simulations to incorporate complexscenarios that are realistic in leading-edge semiconductor fabrication. To the best of our knowledge, there is no published work on full wave analysis of Gaussian beam scattering due to particles/defectson semiconductor wafers. The multi-scale nature of the problem (i.e. a small defect on a large wafer),requires the use of numerical field solvers executed on a multiprocessor cluster to obtain accurate results.Our work stands out from the previously published results in terms of the use of cutting edge modelingresources, better accuracy attributed to full wave methods, Gaussian laser beam excitation instead ofplane wave excitation, shorter wavelength of $193 \mathrm{~nm}$, sub-40 nm defect sizes, and the flexibility to modeldifferent defect shapes, sizes, materials and multilayer substrates.

\section{MODELING FRAMEWORK}

In this work, we use a laser beam of $193 \mathrm{~nm}$ wavelength, a commonly used wavelength in leading semiconductor photolithography systems [9]. The refractive index of silicon at $193 \mathrm{~nm}$ is $\mathrm{n}=0.883+2.778 \mathrm{i}[10]$. The geometry of our light scattering problem is shown in Fig. 1. It consists of a cylindrical Silicon wafer, the top surface of which coincides with the xy plane. A Gaussian beamhaving a minimum beam width (at its waist) of $400 \mathrm{~nm}$ is incident on the silicon wafer at an angle of incidence equalto $30^{\circ}$ with respect to z-axis and the laser beam axis lies on the xz-plane (i.e. wave vector $\hat{k}=0.5 \hat{x}-0.866 \hat{z}$ ). TheGaussian beam focal point location coincides with the coordinate origin and the field amplitude at this point is $1 \mathrm{~V} / \mathrm{m}$. We consider two polarizations in this paper. For perpendicular polarization (s-polarization), the polarization vector is given by $\hat{e}=\hat{y}$. For parallel polarization (p-polarization), the polarizationvector $\hat{e}=$ $0.866 \hat{x}+0.5 \hat{z}$. The parameter of interest for optical detection is the far zone scattered powerdensity. Throughout this paper, we have used $20 \log _{10}\left|\bar{E}_{s c}(r=1 \mathrm{~m}, \theta, \varphi)\right|$ to quantify scattered powerdensity. $\bar{E}_{\mathrm{sc}}(\mathrm{r}=1 \mathrm{~m}, \theta, \varphi)$ is the scattered electric field intensity as a function of polar angle $\theta$ andazimuth angle $\varphi$ on a sphere of radius 1 meter. Since we are interested primarily in the scattered fieldabove the wafer, $\theta$ varies from 0 to 90 degrees. However, $\varphi$ ranges from 0 to 360 degrees. The goal ofour framework is to accurately model scattered light intensity values at all $\theta$ and $\varphi$ angles for a rangeof realistic defects on a semiconductor wafer surface. It should be noted that the defect sizes are in the nanometer range and hence all practical optical 
detectors would be in the far field scattering zone. Hence using a value of $r=1 \mathrm{~m}$ is just a reference value. Once the field at $r=1 \mathrm{~m}$ is known, the fields at any other value of $r$ in the far zone can be calculated.

The diameter of silicon wafers used in modern semiconductor fabrication could be as large as $450 \mathrm{~mm}$. Since this dimension is much larger than the wavelength under consideration, it is impractical toinclude the entire wafer in the simulation. Fortunately, since the Gaussian beam is spatially confined(unlike a plane wave), a smaller local area of the wafer effectively emulates large realistic wafer diameters.By using wafers with progressively increasing radii, it was found that the scattering pattern convergesfor radii greater than $1200 \mathrm{~nm}$. Because of the finite spatial extent of the Gaussian beam, a furtherincrease in the wafer radius did not result in a significant change in the scattering pattern. This wasconfirmed by plotting the magnitude of electric field on the $\varphi=0^{\circ}$ plane vs. the wafer radii used insimulation. A $1600 \mathrm{~nm}$ radius wafer was used for the simulation results shown in this paper.

We used ANSYS HFSS for the simulation of the above mentioned model. A Perfectly Matched Layer (PML) was used to truncate the problem geometry. Gaussian beam excitation was used as the source. The Gaussian beam excitation option in HFSS allows the entry of arbitrary polarization, propagation vector, focal point location and beam width at focal point. Our framework is flexible enough to simulate a wide variety of incident angles, excitation wavelengths, beam widths, incident wave polarizations, defect shapes, defect dimensions, and defect and wafer surface materials.

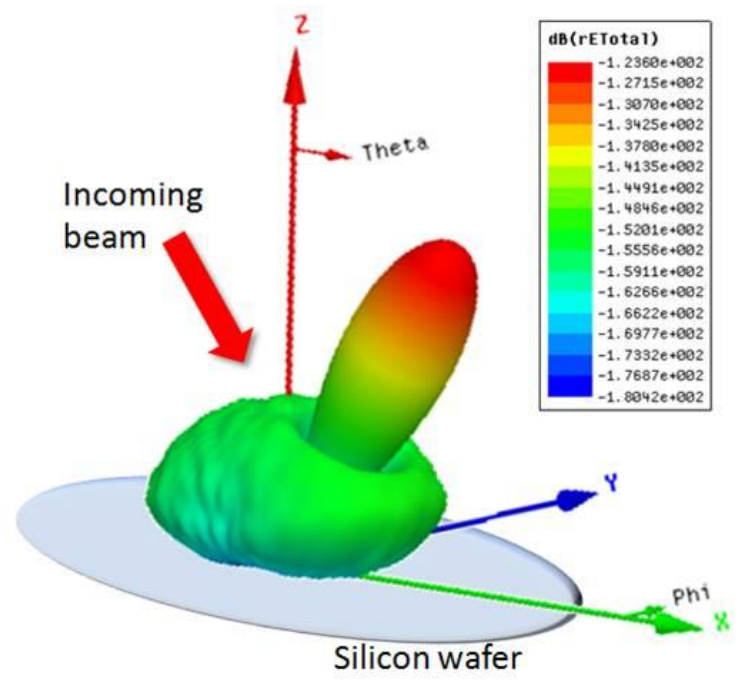

Figure 1. 3D scattering pattern: $40 \mathrm{~nm}$ radius sphere on wafer. The incident beam direction is given by $\hat{k}=$ $0.5 \hat{x}-0.866 \hat{z}$

\section{RESULTS}

We now show scattering patterns for spherical and ellipsoidal defects located on semiconductor wafersfor $\mathrm{s}$ and p-polarizations. For each polarization, we start with a bare wafer (i.e. a wafer having nodefects) to determine the noise floor of our model. This is followed by simulations of $20 \mathrm{~nm}, 30 \mathrm{~nm}$ and $40 \mathrm{~nm}$ radii silicon nanospheres located on the silicon wafer. Finally, we show simulations of a nanometerscale ellipsoidal particles located on a silicon wafer at $0^{\circ}, 45^{\circ}, 90^{\circ}$ and $135^{\circ}$ orientation angles. 


\subsection{Base Si wafer}

When a bare wafer is illuminated with a Gaussian beam, the beam undergoes specular reflection. Thespecularly reflected beam propagates at $\theta=30^{\circ}$ and $\varphi=0^{\circ}$. In addition, weak scattering is observedat non-specular directions. Figure 2(a) shows the scattering pattern of the bare wafer for s-polarizedillumination. This figure is a two-dimensional (2D) plot of $20 \log _{10} \mid \overline{\mathrm{E}}_{\mathrm{sc}}(\mathrm{r}=$ $1 \mathrm{~m}, \theta, \varphi) \mid$ as a function of $\theta$ and $\varphi$. The specularly reflected beam can be observed in the range $\theta \in\left[10^{\circ}, 50^{\circ}\right]$ and in the vicinity of $\varphi=0^{\circ}$. For s-polarization, the peak of the specularly reflected beam is $-122 \mathrm{~dB}$, and the maximum valueof the scattered field outside this specularly reflected beam is $-170 \mathrm{~dB}$. Figure 3(a) shows the scatteringpattern of the bare wafer for ppolarized illumination. For the case of p-polarization, the peak of thespecularly reflected beam is $-127 \mathrm{~dB}$, and the maximum value of the scattered field outside this specularbeam is $-171 \mathrm{~dB}$. Accordingly, the computational noise floor of our model is $-170 \mathrm{~dB}$ for s-polarizationand -171 $\mathrm{dB}$ for p-polarization. Scattered light intensity from a defect needs to exceed this noise floorfor the defect to be able to be detected reliably. It should be stated that in the $2 \mathrm{D}$ plots, the highestvalue of scattered field is truncated to $-150 \mathrm{~dB}$ for visual clarity.

\subsection{Silicon sphere on Silicon wafer: s-polarization}

Figures 2 (b-d) show scattering patterns of silicon spheres with radii $20 \mathrm{~nm}, 30 \mathrm{~nm}$ and $40 \mathrm{~nm}$ locatedon the wafer surface for the case of s-polarized Gaussian beam excitation. Figure 1 is in fact a three-dimensional view of figure 2(d). In the 2D plots, the specular reflection is split into two halves along the $\varphi=0^{0}, 360^{\circ}$ planes. This is because of the symmetry of the incident illumination beam with respect toxz plane. Consequently, the specularly reflected beam is also symmetric with respect to the $\mathrm{xz}$ plane.The maximum values of scattered radiation outside the specularly reflected beam are $-164 \mathrm{~dB},-159 \mathrm{~dB}$, and $-154 \mathrm{~dB}$ for the $20 \mathrm{~nm}, 30 \mathrm{~nm}$ and $40 \mathrm{~nm}$ spheres, respectively. It should be noted that forevery $10 \mathrm{~nm}$ decrease in sphere radius, the maximum scattered field gets reduced by $5 \mathrm{~dB}$. We find that scattering pattern is below the noise floor for spheres having a radius less than $10 \mathrm{~nm}$ with an s-polarized incident beam. The spolarized incident beam induces an electric dipole oriented along they direction. The radiation pattern of a dipole is dough-nut shaped with its null along its direction ofpolarization. These nulls can be seen in Figures $2(\mathrm{a}-\mathrm{d})$, along $\varphi=90^{\circ}, 270^{\circ} ; \theta=90^{\circ}$. The green ellipsein figure 2(d) denotes the null along $\varphi=270^{\circ}$. As the radius of the sphere increases, the scatteringpattern becomes asymmetric with more scattering along the forward direction (i.e. $\varphi=$ $\left.0^{0}\right)$. 

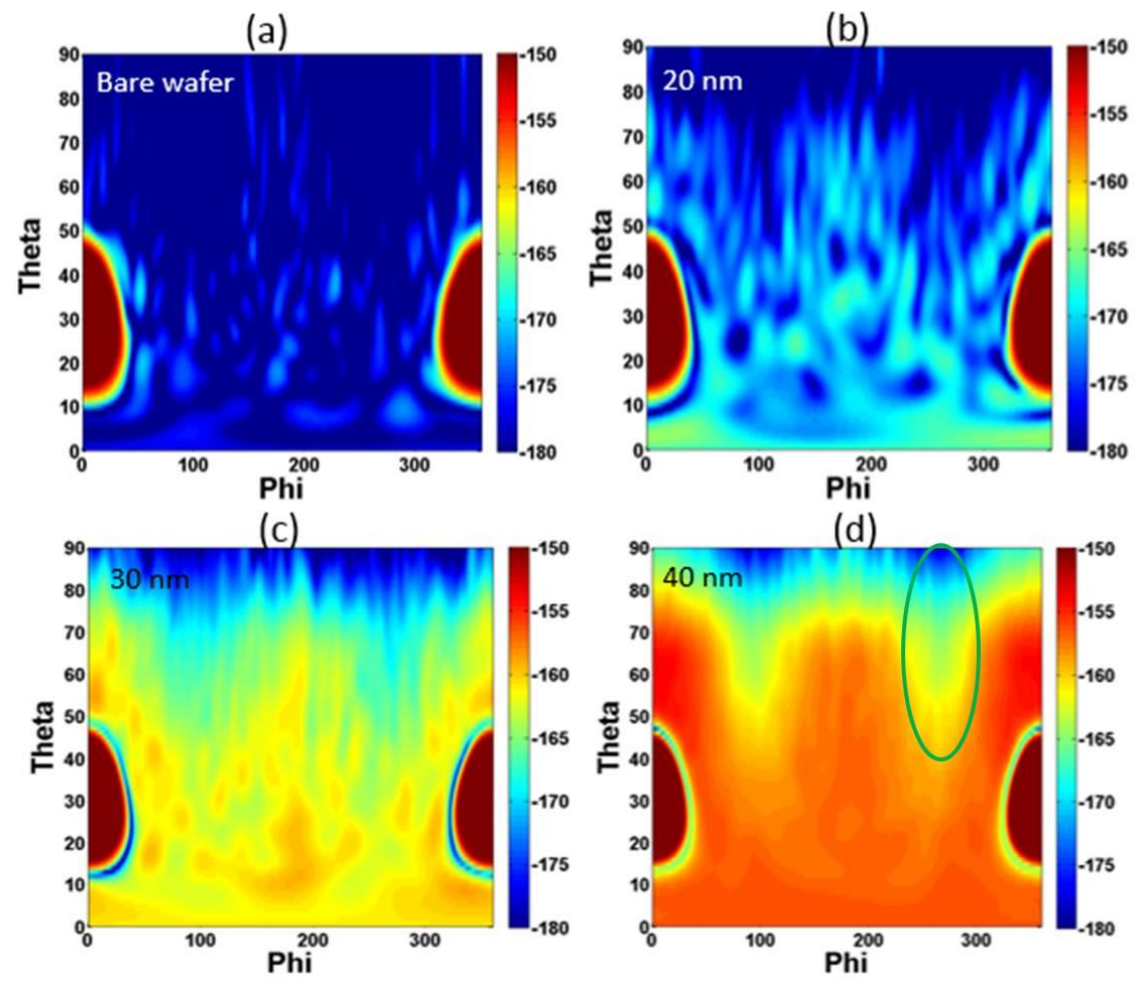

Figure 2. Light scattering by a) bare Si wafer, b) $20 \mathrm{~nm}$ sphere on a Si wafer, c) $30 \mathrm{~nm}$ sphere on aSi wafer and d) $40 \mathrm{~nm}$ sphere on a Si wafer, all of which are illuminated with a s-polarized Gaussianbeam having a wavelength of $193 \mathrm{~nm}$.

\subsection{Silicon sphere on Silicon wafer: p-polarization}

Figures 3 (b-d) show scattering pattern for silicon spheres with radii $20 \mathrm{~nm}, 30 \mathrm{~nm}$ and $40 \mathrm{~nm}$ located on the surface of a silicon wafer for the case of p-polarized Gaussian beam excitation. The maximum values of scattered radiation excluding the specularly reflected beam are given by $-169 \mathrm{~dB},-162 \mathrm{~dB}$ and $-156 \mathrm{~dB}$ for the $20 \mathrm{~nm}, 30 \mathrm{~nm}$ and $40 \mathrm{~nm}$ spheres, respectively. We observe that the scattered energy in the case of p-polarization is less than that of s-polarization. We find that scattering pattern is below the noise floor for spheres having a radius less than $20 \mathrm{~nm}$ with a p-polarized incident beam. This is in contrast to $10 \mathrm{~nm}$ detection limit for s-polarization. Figure 4 shows a comparison of scattering patterns with $\mathrm{s}$ and p polarization incident beams for a $40 \mathrm{~nm}$ sphere on silicon wafer. Figure 4(a) illustrates this comparisonin the $\varphi=0^{0}$ plane, and Figure 4(b) illustrates this comparison in the $\varphi=90^{\circ}$. Figure 4(a) shows that the scattered intensity for s-polarization is higher than the scattered intensity of p-polarization in the $\varphi=0^{0}$ plane. On the other hand, Figure 4(b) shows that the scattered intensity for s-polarization rolls off with respect to azimuth angle at a faster rate than p-polarization. These observations can be explained by the rotation of the sphere scattering pattern when incident polarization is rotated from $\mathrm{s}$ to $\mathrm{p}$. Because of the decreased scattered field intensity, s-polarization is preferred over p-polarization in industrial wafer inspection systems.

\subsection{Silicon ellipsoid on Silicon wafer: s-polarization}

We now show the scattering pattern of an ellipsoidal particle with axis lengths $140 \mathrm{~nm}$ x $60 \mathrm{~nm} \times$ $60 \mathrm{~nm}$ located on a silicon wafer. The incident beam is s-polarized (i.e. $\hat{e}=\hat{y}$ ). Four different orientationangles $0^{\circ}, 45^{\circ}, 90^{\circ}$ and $135^{\circ}$ are used. Orientation angle denotes the angle between the major axis ofthe ellipsoid and the $\mathrm{x}$-axis. For example, for the $45^{\circ}$ case, the $140 \mathrm{~nm}$ length axis of 
ellipsoid makesan angle of $45^{\circ}$ with $\mathrm{x}$-axis. The major axis of the ellipsoid is parallel to the plane of the silicon waferin all four orientation angle cases. Figure 5 shows the scattering pattern for the four cases. We observethat the scattering pattern for the ellipsoid with $90^{\circ}$ orientation, shown in Figure 5(d), is similar to thescattering pattern of a $40 \mathrm{~nm}$ radius sphere shown in Figure 2(d). This is because of a couple of reasons:1) volume of the $40 \mathrm{~nm}$ radius sphere is roughly equal to the volume of the ellipsoid; 2) major axis ofellipsoid with $90^{\circ}$ orientation is along the direction of incident beam polarization. From Figure 5, it canbe observed that nulls occur along the direction of the ellipsoid major axis away from the specularlyreflected lobe $\left(\varphi=0^{0}, 360^{\circ}\right)$. For instance, when the orientation angle is $45^{\circ}$ the major axis lies along $\varphi=45^{\circ}, 225^{\circ}$ and the null occurs at $\varphi=225^{\circ}$. A similar observation can be made in Figure $5(\mathrm{c})$ forthe case of $135^{\circ}$ orientation angle.

\section{CONCLUSIONS}

This paper provides accurate full wave simulation results of laser beam scattering of spherical and ellipsoidal Silicon particles located on a cylindrical silicon wafer. The results presented in this paper is as close as to real world measurement scenario when compared to previous published results. Thescattering pattern of a defect is found to have a close dependence on the shape of the defect. Fors-polarization, the maximum scattered field outside the specularly reflected lobe reduces by $5 \mathrm{~dB}$ for every $10 \mathrm{~nm}$ reduction in sphere radius. Such a dependence can be used to detect the size of the defect. For p-polarization, the scattered energy is less than that of s-polarization. For the case of ellipsoidal defects, the scattering pattern exhibits a rotation with respect to the orientation of the major axis of the ellipsoid. Wafer surface roughness can have a profound effect on the scattering pattern and ultimately determines the smallest defect that can be detected. Although we ignore roughness in this paper, defect scattering pattern by itself could help us build more sensitive instruments. We believe the results inthis work will be invaluable to wafer inspection technology companies, who can use these data to designnovel instruments as well validate their numerical models [11]. Future work will include scattering study ofmore complicated defects such as Crystal Originated Pits (COPs) and different materials. 
(a)

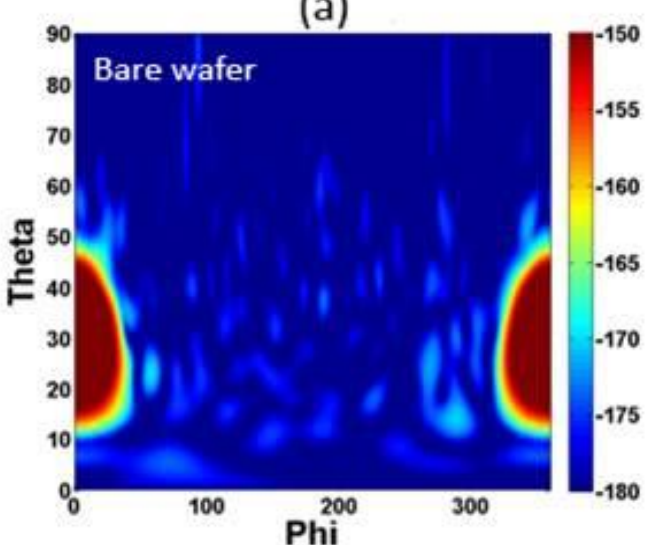

(c)

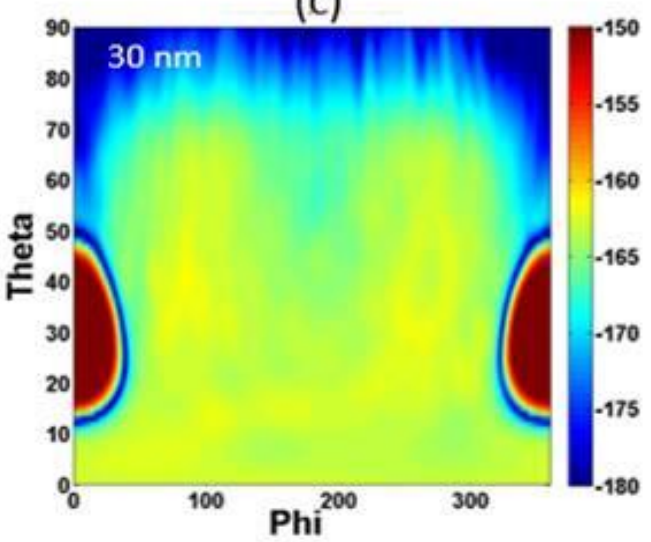

(b)

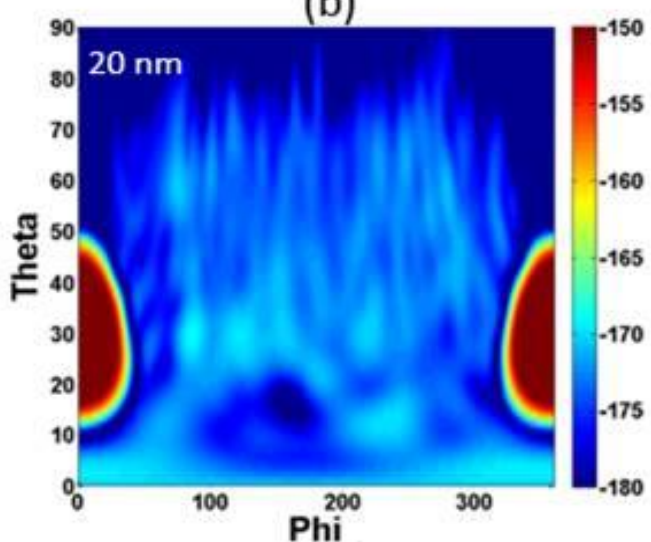

(d)

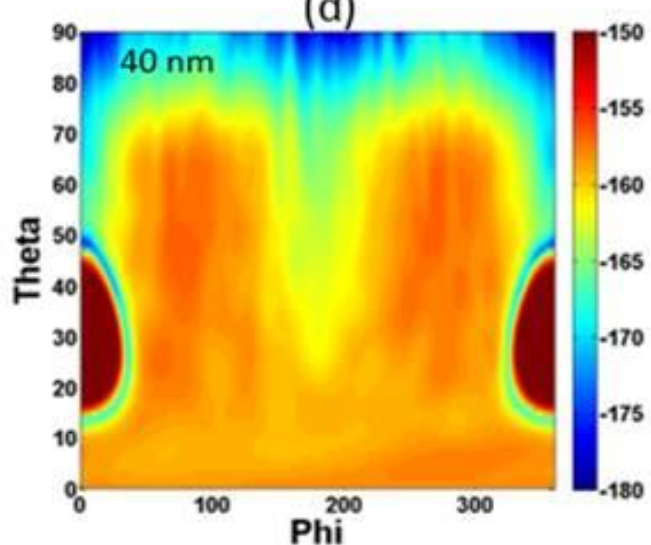

Figure 3. Light scattering by a) bare Si wafer, b) $20 \mathrm{~nm}$ sphere on a Si wafer, c) $30 \mathrm{~nm}$ sphere on aSi wafer and d) $40 \mathrm{~nm}$ sphere on a Si wafer, all of which are illuminated with a p-polarized Gaussianbeam having a wavelength of $193 \mathrm{~nm}$.

a

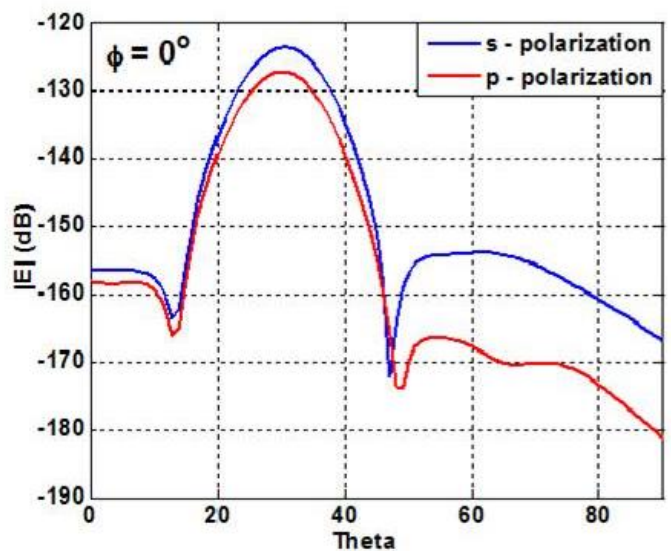

b

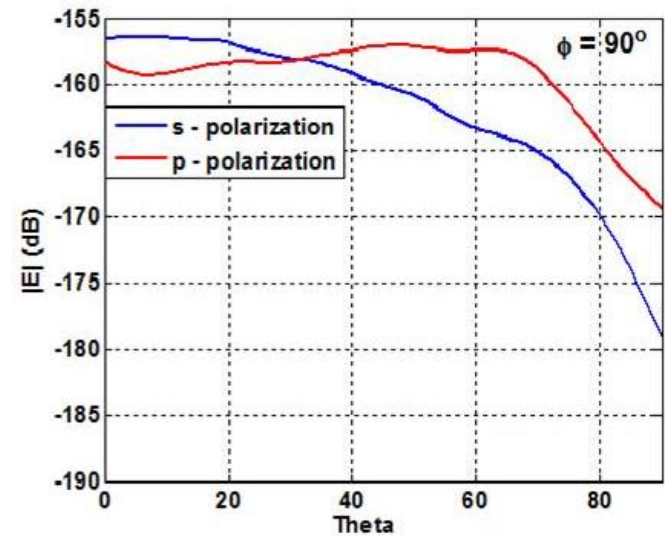

Figure 4. a) Scattering pattern $\left(\varphi=0^{0}\right)$ comparison for $40 \mathrm{~nm}$ radius sphere on wafer: $\mathrm{s}$ vs ppolarization b) Scattering pattern $\left(\varphi=90^{\circ}\right)$ comparison for $40 \mathrm{~nm}$ radius sphere on wafer: $\mathrm{s}$ vs ppolarization. 

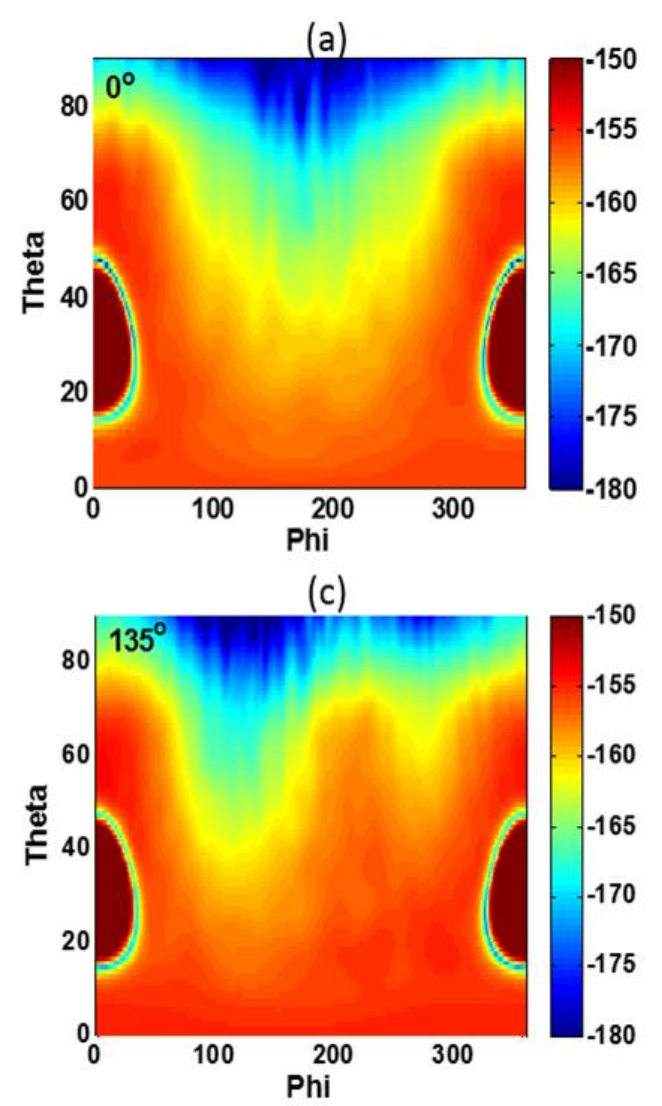
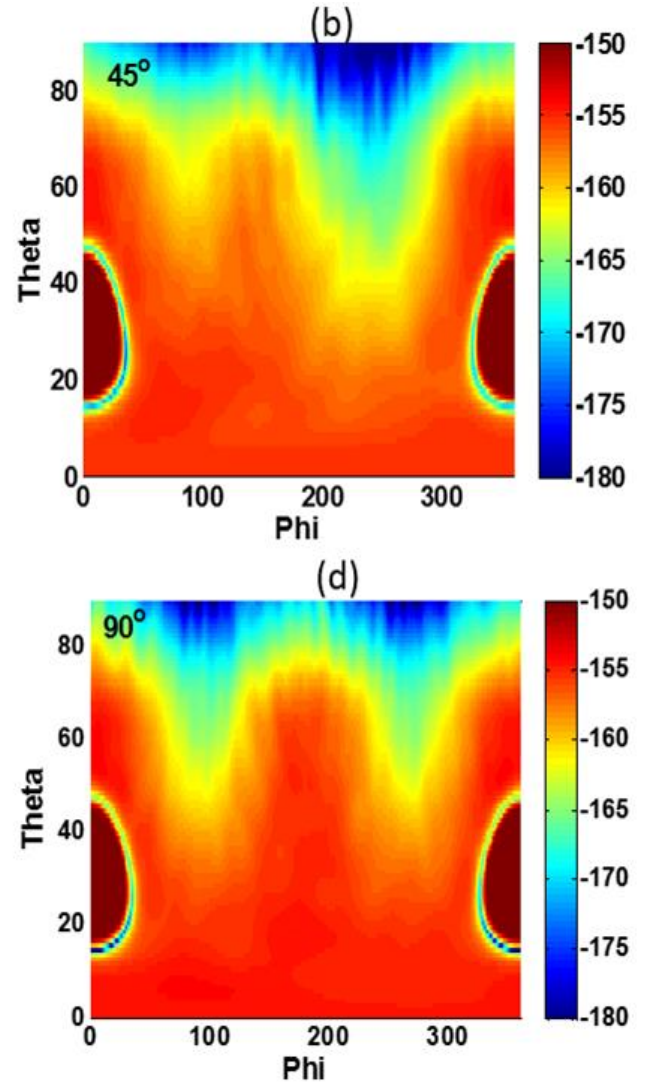

Figure 5. Light scattering by a) bare Si wafer, b) $20 \mathrm{~nm}$ sphere on a Si wafer, c) $30 \mathrm{~nm}$ sphere on aSi wafer and d) $40 \mathrm{~nm}$ sphere on a Si wafer, all of which are illuminated with a p-polarized Gaussianbeam having a wavelength of $193 \mathrm{~nm}$.

\section{REFERENCES}

[1] Gargini, P., "The International Technology Roadmap for Semiconductors (ITRS): Past, present and future" GaAs IC Symposium, 2000. 22nd Annual , 3-5, 2000.

[2] Isogai, S., Endo, F., Kobayashi, K., and Arai, K., "Hitachi Review: Advanced Defect Review System for Yield Enhancement (RS Series)", 2007.

[3] Feng Y., and Burkett S.L.,Fabrication and Electrical Performance of Through Silicon Via Interconnects Filled with a Copper/Carbon Nanotube Composite; Journal of Vacuum Science and ; Technology B. 33(2): 022004 (2015)

[4] Strover, J. C. Optical Scattering: Measurement and Analysis, $3^{\text {rd }}$ edition, SPIE Press 2012.

[5] Nebeker, B. M., and Hirleman, E. D Light scattering from microstructures, Springer, 2000.

[6] Bohren, C. F., and Huffman, D. R Absorption and Scattering of Light by Small Particles, Wiley$\mathrm{VCH}, 1998$

[7] Taubenblatt, M., and Tran, T. K "Calculation of light scattering from particles and structures ona surface by the coupled-dipole method" J Opt Soc Am, 1993.

[8] Kim, J. H., Ehrman, S. H., Mulholland, G. W , and Germer, T. A., "'Polarized light scattering by dielectric and metallic spheres on oxidized silicon surfaces," Appl. Opt. 43 , 585-591 (2004)

[9] Zant, P. V., "Microchip Fabrication: A Practical Guide to Semiconductor Processing", McGrawHill, 2014

[10] Palik, E. D., "Handbook of Optical Constants of Solids", Elsevier Inc, 1997

[11] Tuyen K. Tran, "Defect Inspection for Advanced Technology Nodes", International Conference on Frontiers of Characterization and Metrology for Nanoelectronics.2015 http://www.nist.gov/pml/div683/conference/upload/tran.pdf 


\section{Authors}

Srikumar Sandeep received his $\mathrm{MS}$ and $\mathrm{PhD}$ in electrical engineering from University of Colorado, Boulder in 2011 and 2012 respectively. From 2012 to 2013, he was a postdoctoral research associate at University of Colorado, Boulder. Presently, he is a postdoctoral researcher at Ecole Polytechnique, Montreal. He has more than 5 years of industrial experience in several fields of electrical engineering such as computational electromagnetics, signal integrity, embedded systems and software development. He is a co-inventor of 2 US patents. His technical interests include computational and applied electromagnetics.

Alexander Kokhanovsky graduated from the Department of Physics at the Belarusian State University, Minsk, Belarus in 1983. He received his Ph.D in Optical Sciences from the B.I. Stepanov Institute of Physics, National Academy of Sciences of Belarus in 1991. His habilitation work (Main Geophysical Observatory, St.Petersburg, Russia, 2011) was devoted to the development of advanced cloud and snow remote sensing techniques based on spaceborne observations. His research interests include studies on light propagation and scattering in terrestrial atmosphere. Dr. Kokhanosky is the author of books Light Scattering Media Optics: Problems and Solutions, Polarization Optics of Random Media, Cloud Optics and Aerosol Optics. He has published more than 300 papers in the field of environmental optics, radiative transfer and light scattering
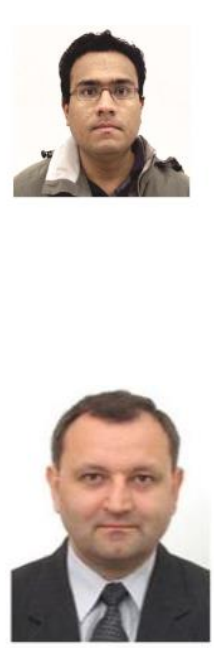